\title{
Design of Safety Early Warning Based on Ultrasound
}

\author{
Kai Xia ${ }^{*}$ and Ming Yang \\ School of Electronics Engineering, Yangtze Normal University Fuling Chongqing China 408100 \\ ${ }^{*}$ Corresponding author
}

\begin{abstract}
A safety driving warning system which can use Ultrasonic wave to measure the distance and generate an early warning of dangerous distance has been designed in order to improve driving safety by the propagation velocity of ultrasonic wave in the air soon, less energy consumption, directional well. STC89C52 MCU is employed as the key control unit; HC-SR04 ultrasonic module is utilized to complete ranging and speed detection; and temperature sensors are used to reduce the influence of temperature in sonic ware; Through displaying the measured speed, distance, and in the display module of LCD 1602 screen, the speed and distance will be measured and compared so as to improve measurement precision; when the distance is less than security distance, the buzzer will utter an alarm to remind the driver to react properly.
\end{abstract}

Keywords-mcu; ultrasound; measured speed; measured distance

\section{INTRODUCTION}

Society has been in constant development and progress, people's living standard has been varying degrees of increase, China's car ownership continuously increase year by year, lead to all kinds of traffic accidents frequently occur, mainly car crash, so safety problem has become a major topic to be solved. Special European scientists have done a study: the driver can be within 0.5 seconds before the dangerous accident get warnings, $60 \%$ to avoid collision, $30 \%$ of head-on crash and $50 \%$ of road accidents. Nowadays the traffic accidents has been a serious threat to people's personal safety, the related security technology research also more and more get the attention of people ${ }^{[1]}$. Using ultrasonic ranging, it's less affected by the environment, the accuracy is higher and the operation is simple, the cost is low, can meet the design requirements. So ultrasonic ranging has great practical significance, believe that the further development of ultrasonic ranging will continue in the future.

\section{ULTRASONIC RANGING}

Ultrasonic distance measurement based on ultrasonic wave reflected back from the launch to detect obstacles of time lag, the distance between the objects are obtained. Ultrasonic transmitting terminal to a fixed direction of launch ultrasonic wave, and start the timer, after ultrasonic encounter obstacles will be reflected back, when ultrasonic receiver receives is reflected ultrasonic wave, stop the timer. Can know the time of the ultrasonic wave to travel between objects, ultrasonic velocity in the medium is known, so the distance between the object can be obtained. Compared with other sensors, ultrasonic sensor has many advantages, its directivity is strong, the energy loss in the process of spread slowly, in the medium transmission distance, is not restricted by environment [2].

Made by single chip microcomputer, a pin a $40 \mathrm{KHz}$ pulse signal, make the ultrasonic wave emit ultrasonic module to a certain direction, at the same time open MCU internal timer, when the ultrasonic module receives the reflected back after meet obstacles of ultrasonic, stop the timer, then will be sent to the single chip microcomputer internal timer record time $\Delta t 1$ for data processing, through the formula to calculate the distance $\mathrm{s} 1$. After receiving the echo of launch ultrasonic wave again, the same time $\Delta \mathrm{t} 2$ interval for data processing, calculate the distance $\mathrm{s} 2$ the velocity of an object as

$$
\mathrm{v}=\frac{2\left(s_{1}-s_{2}\right)}{\Delta t_{1}+\Delta t_{2}}
$$

As car brake depends mainly on the ground and the friction between the tires, assuming that the friction coefficient is $\mu$, auto speed $\mathrm{v}$, the acceleration of gravity $\mathrm{g}$, the braking distance, so the car braking distance and speed is proportional to the square

$$
\frac{v^{2}}{2 \mu g}
$$

When the friction coefficient of a certain distance depends on the speed of the brakes. Generally 0.8 value of friction coefficient, can be concluded that the speed and braking distance relationship, as shown in table 1 .

\section{TABLE I. SPEED AND BRAKING DISTANCE}

\begin{tabular}{|c|c|c|c|c|c|c|c|c|}
\hline $\begin{array}{c}\text { speed of the } \\
\text { car(Km/h) }\end{array}$ & 20 & 30 & 50 & 60 & 70 & 80 & 90 & $\begin{array}{c}10 \\
0\end{array}$ \\
\hline braking distance (m) & 2.0 & 4.4 & $\begin{array}{c}12 . \\
3\end{array}$ & $\begin{array}{c}17 . \\
7\end{array}$ & $\begin{array}{c}24 . \\
1\end{array}$ & $\begin{array}{c}31 . \\
5\end{array}$ & $\begin{array}{c}39 . \\
7\end{array}$ & $\begin{array}{c}49 . \\
2\end{array}$ \\
\hline
\end{tabular}

Ultrasonic is a kind of mechanical wave, it's generally more than $20 \mathrm{KHz}$ frequency, it can be directed straight lines in a homogeneous medium, therefore it has good beam and directional[1].. Range speed measuring principle as follows, ultrasonic sensor transmitter launch a bundle of $40 \mathrm{KHz}$ sound waves, at the same time start the MCU internal timer, ultrasonic bounce back after meet obstacles, reflected wave received by ultrasonic sensor at the receiving end and stop the timer, back and forth for ultrasonic time, the set time $t$, the ultrasonic velocity in air for $\mathrm{c}$, using the formula $\mathrm{D}=(\mathrm{ct}) / 2$, calculate the distance. After receiving ultrasonic echo, ultrasonic launch again, to send and receive time interval and calculate the distance, the object can be obtained velocity $=(2$ twice distance difference)/time interval and twice. Ultrasonic wave propagation velocity and temperature relations as shown in table 2. 
TABLE II. ULTRASONIC WAVE PROPAGATION VELOCITY AND TEMPERATURE

\begin{tabular}{|c|c|c|c|c|c|c|c|c|c|c|c|}
\hline project & \multicolumn{9}{|c|}{ numerical } \\
\hline $\begin{array}{c}\text { temperat } \\
\text { ure }\end{array}$ & -30 & -20 & -10 & 0 & 10 & 20 & 30 & 40 & 50 & 60 & 100 \\
\hline $\begin{array}{c}\text { speed of } \\
\text { sound(m/ } \\
\text { s) }\end{array}$ & 313 & 319 & 325 & 332 & 338 & 344 & 350 & 356 & 361 & 367 & 388 \\
\hline
\end{tabular}

Design uses the temperature sensor DS18B20 environment $\mathrm{t}$, therefore, for temperature compensation circuit, namely:

$$
v=v_{0} \sqrt{1+\frac{t}{273.15}}
$$

Including $=331.5 \mathrm{~m} / \mathrm{s}$ to $0{ }^{\circ} \mathrm{C}$ when the speed of sound [1], a formula can be $\mathrm{c}=331.5+0.607 \mathrm{t}$, according to the formula, we can get the current temperature wave velocity.

Speed using time difference method, it is assumed that the first ultrasound in time $\Delta \mathrm{t} 1$ for back and forth between the object and receives the reflected ultrasonic wave launch ultrasonic signal again, after a second time $\Delta \mathrm{t} 2$ interval for back and forth. The measured distance $\mathrm{s} 1$ for the first time, second time measured distance is $\mathrm{s} 2$, the rate of movement of the object as shown use 1 [4]:

Ultrasonic module pin connected to VCC respectively, $\mathrm{P} 1.0$, P1.1 \& GND. Module through the SCM P1.0 port to ultrasonic module 3 mouth TRIG a high level of at least $10 \mu$ s trigger signal, the ultrasonic module will automatically send eight $40 \mathrm{KHz}$ square wave, and the automatic detection of echo signal, echo, if detected by mouth ECH0 output to MCU P1.1 port 2 is a high level, maintain the length of the high level, is the time of ultrasonic wave back and forth for distance measurement. Test the distance $=($ the speed of sound $*$ high level time $) / 2$ [4].

The design USES DS18B20 temperature sensor. The sensor support "1-wire bus" interface. Measuring range - $55{ }^{\circ} \mathrm{C} \sim+$ $125{ }^{\circ} \mathrm{C}$, in $-10{ }^{\circ} \mathrm{C} \sim+85^{\circ} \mathrm{C}$ range, precision of $0.5{ }^{\circ} \mathrm{C}$, the single chip microcomputer through an I/O port with DS18B20 link, which can realize the two-way communication between the two [5]. The output data for nine signed binary number, the need for temperature calibration and linear correction, make the procedure of preparation was simplified, so widely used [6]

\section{The Software System IS DESIGNED}

The main program is mainly responsible for complete the initialization and ultrasonic distance and velocity measurement, and through the LCD display module display the measured value, calculated, through the single chip microcomputer internal buzzer alarm when the minimum safety distance. By single chip microcomputer P1.0 pin to launch a high level for the ultrasonic module, drive module launch ultrasonic, close the external interrupt at the same time, start the timer, when receives the echo open external interrupt, off timer stop timing, then read time value $t$, and start measuring temperature subroutine, measuring the temperature of the current environment, accurately compute the current velocity $\mathrm{c}$, then back to the single chip microcomputer to calculate the distance inside, complete a measurement of launch ultrasonic wave again, after calculate the second distance, time difference method is used for object movement speed. Through single chip microcomputer to calculate the safe distance under the current speed, the buzzer alarm when less than the safe driving distance.

\section{System DeBUGGING}

After checking the hardware system, gradually can check each subroutine functions correctly. Because in the program, is separate modules, so conducive to subsequent inspection and modification. Through test, the main program can transmit and receive ultrasonic correctly, LCD module can show distance, velocity and temperature numerical, temperature measurement module can real-time measuring temperature, buzzer alarm when less than the safe distance. As shown in figure 1 (a), after the system is powered on, LCD display the upper left shows real-time measuring distance, display the current speed at the upper right, every $0.1 \mathrm{~s}$ test, a distance below the current environment temperature, used for temperature compensation.

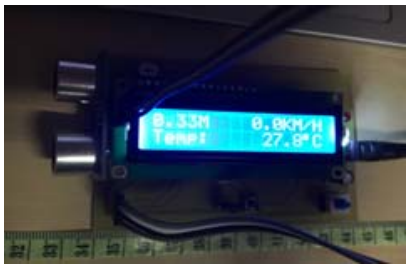

(a)

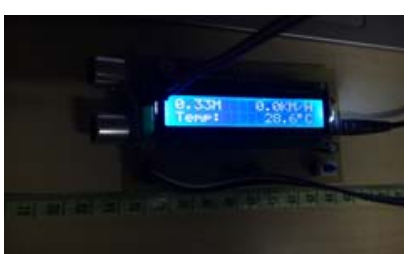

(b)

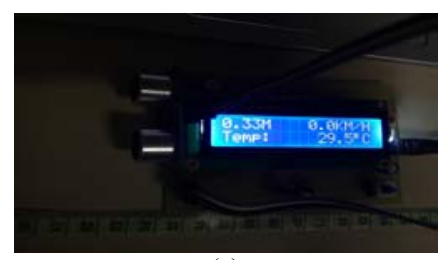

(c)

\section{FIGURE I. TEMPERATURE COMPENSATION}

As shown in figure 2 (a), (b), (c). In different environmental temperature by detecting distance, to verify the accuracy of the temperature compensation, the experiment respectively in $27.8^{\circ} \mathrm{C}$ and $28.6{ }^{\circ} \mathrm{C}$ and $29.5{ }^{\circ} \mathrm{C}$ three temperature, detection distance, and through scale, verify its correctness. Table 3 shows the three temperature measuring distance and the actual distance.

Experiments show that this design, the success of the temperature compensation, and reached the design requirements. 
TABLE III. TEMPERATURE COMPENSATION

\begin{tabular}{|c|c|c|c|}
\hline $\begin{array}{c}\text { environment } \\
\text { temperature }\left({ }^{\circ} \mathbf{C}\right)\end{array}$ & $27.8^{\circ} \mathrm{C}$ & $28.6^{\circ} \mathrm{C}$ & $29.5^{\circ} \mathrm{C}$ \\
\hline $\begin{array}{c}\text { measured } \\
\text { distance(m) }\end{array}$ & 0.33 & 0.33 & 0.33 \\
\hline actual distance(m) & 0.33 & 0.33 & 0.33 \\
\hline
\end{tabular}

After completion, validation of temperature compensation for alarm, when the movement speed of $0.3 \mathrm{~km} / \mathrm{h}$, the minimum safe distance should be 0.3 meters, therefore, in the distance less than or equal to $0.3 \mathrm{~m}$, the buzzer will alarm, alarm prompt light leds will be on the red light. As shown in figure 2 (a), (b), (c).

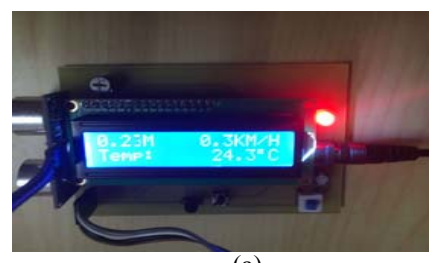

(a)

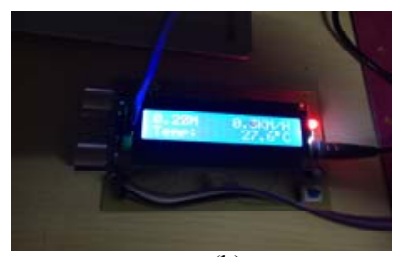

(b)

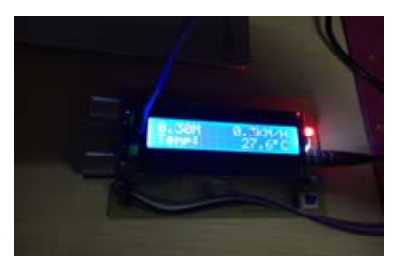

(c)

FIGURE II. ALARM SYSTEM VERIFICATION

Experiment proves that the speed of $0.3 \mathrm{~km} / \mathrm{h}$, when the distance of $0.3 \mathrm{~m}$, was carried out on the correct system alarm, when the distance of $0.28 \mathrm{~m}$ and $0.23 \mathrm{~m}$, have the right to the alarm, so the writing of the program is correct, successfully completed the alarm, has reached the design requirements.

\section{SUMMARY}

Design by using ultrasonic transmission speed in the air, less energy consumption, good directivity, so accept the reflected the characteristics of the echo is relatively complete, realize the distance work, complete the dangerous distance warning safety alarm system, in order to improve road safety.

\section{REFERENCE}

[1] Hu Rui Zhou Xiqing. Range alarm system based on ultrasonic sensor design [J]. Science, 2009, (7): pp. 58, 12.

[2] Wu Chao, ya-wen Dai. The design of the ultrasonic ranging system based on AT89S52 single chip microcomputer [J]. Journal of zhongyuan institute of technology, 2008, 12 (5): pp. 65-68.

[3] Shen Yan, xiao-rong Gao etc. Ultrasonic range finder based on singlechip design [J]. Journal of modern electronic technology, 2012, 35 (7): pp. 126-129.

[4] LanYu. Instrumentation amplifier applications in laser heterodyne glass thickness measurement system $[\mathrm{J}]$. Journal of foreign electronic measurement technology, 2012, 31 (3): pp.79-82.

[5] Guo Qing. Based on the ultrasonic ranging STC89C52 anti-collision system design [J]. Instrument technique and sensor, 2011, (6): pp. 74-77.

[6] Zhang Jun. Intelligent temperature sensor DS18B20 and its application [J]. Instrument technology, 2010, (4): pp.68-70. 